\title{
Investigating the construct validity of Sternberg's triarchic abilities test level-H (Arabic version)
}

\author{
Rana TH. Momani *, Salem A. Gharaibeh \\ Psychology Department, Qassim University, Buraideh, Saudi Arabia
}

\section{A R T ICLE IN F O}

\section{Article history:}

Received 1 June 2017

Received in revised form

13 September 2017

Accepted 17 September 2017

\section{Keywords:}

Triarchic intelligence theory

Construct validity

Sternberg

STAT-H

\begin{abstract}
A B S T R A C T
Intelligence was traditionally considered as a unitary concept until Robert Sternberg proposed his triarchic intelligence theory and developed the Sternberg Triarchic Abilities Test (STAT) to measure triarchic intelligence. The present study aimed at investigating the construct validity of the Arabic version of STAT level-H by comparing different theoretical models as proposed by Sternberg. Results of the correlation analysis showed insignificant correlations between three aspects of intelligence, namely analytical, practical, and creative abilities. Confirmatory factor analysis results confirmed the structural validity of STAT, as the model of three uncorrelated abilities was deemed as the best fit. The model of general intelligence was insufficient to consider STAT as a measure of general intelligence (g). The current results support Sternberg's results, while many other researchers' findings claimed that STAT is just a measure of (g).
\end{abstract}

(C) 2017 The Authors. Published by IASE. This is an open access article under the CC BY-NC-ND license (http://creativecommons.org/licenses/by-nc-nd/4.0/).

\section{Introduction}

Sternberg triarchic Abilities Test (STAT) is one of the most popular triarchic intelligence tests in the Arab world. Although it is valid in various cultures, there is insufficient evidence for its construct validity in the Arab culture. Sternberg and Grigorenko (2006) stated that triarchic intelligence is individuals' capacity to achieve their ambitions within their sociocultural context through an admixture of adapting to, shaping, and selecting environments. Berry (1974) stated that individuals might be recognized as "intelligent" in a particular culture, but not in other cultures. For example, Ruzgis and Grigorenko (1994) reported that the concept of intelligence in the African communities is different according to their life conditions and environments. Meanwhile, Yang and Sternberg (1997) posited that the concept of intelligence differs between the East and the West. In response to this, the present study aimed to determine the validity of STAT as a measure of triarchic intelligence within the Arab culture.

Sternberg's theory, which is also known as "successful intelligence", and the Sternberg triarchic abilities test (STAT) have been criticized by some

\footnotetext{
* Corresponding Author.

Email Address: rana.momani@yahoo.com (R. T. H. Momani) https://doi.org/10.21833/ijaas.2017.011.005

2313-626X/C 2017 The Authors. Published by IASE.

This is an open access article under the CC BY-NC-ND license

(http://creativecommons.org/licenses/by-nc-nd/4.0/)
}

researchers who claimed that Sternberg's intelligence theory does not differ from the conventional general intelligence (Brody, 2001; Chooi et al., 2014; Gottfredson, 2003).

Meanwhile, many studies which investigated the triarchic intelligence theory using STAT, confirmed the existence of triarchic intelligence as a distinct concept to "g". Although many researchers believe that intelligence entails under a general factor " $g$ ", triarchic intelligence differs from general intelligence (Sternberg, 1997; Sternberg et al., 2000).

Sternberg's triarchic theory has three main aspects, which are analytical, practical, and creative intelligence. Analytical intelligence is required for solving problems and judging the quality of ideas. Practical intelligence refers to the extent of how ideas are used and analyzed in an effective way in individuals' daily lives. Creative intelligence is required for formulating good solutions to problems (Kaufman and Singer, 2004). The primary difference between triarchic intelligence theory and the traditional concept of intelligence is that the latter assumes intelligence revolves around one factor of general mental ability, which is denoted as " $\mathrm{g}$ " (Ekinci, 2014).

Many researchers are interested in the validity of the triarchic intelligence theory and whether it differs from the general concept of mental ability. Sternberg et al. (1999) studied the internal validity of triarchic intelligence theory by administrating the STAT among 326 American high school students. Confirmatory factor analysis (CFA) yielded weak 
correlations between test dimensions, as well as the analytical, practical, and creative abilities .This analysis has also proven that these three abilities have separate structures which support the triarchic theory. It is worth mentioning that previous researchers have linked the weak correlation between "triarchic abilities" and the "inclusion of essay tests in STAT". They have also claimed that essay-type items might not be the best choice to measure triarchic abilities. Grigorenko and Sternberg (2001) investigated the construct validity of the STAT among 511 Russian students, 490 mothers, and 328 fathers of these children. Results of the exploratory principal component analysis showed similar factor structures for these three samples, which confirmed the triarchic theory of intelligence.

Sternberg et al. (2001a) investigated the internal validity of triarchic intelligence theory using STAT among 3,252 students from the U.S., Finland, and Spain. They eliminated the essay test from the original STAT, and subsequently investigated five different models of intelligence. Results of the confirmatory factor analysis supported the triarchic intelligence model with intercorrelations among the triarchic abilities. Nonetheless, the general factor intelligence models showed a poor fit.

Brody (2003) further investigated published studies that dealt with STAT by Sternberg et al. (2001a). Brody (2003) used the original version of the STAT on 199 students who participated in a summer school program at Yale. The relationships between triarchic abilities with other popular tests and academic achievement, as well as the relationships between triarchic abilities among themselves were studied. The results showed the existence of a general variance in three different analyses, namely CFA, correlation, and principal component analysis.

Chooi et al. (2014) published an article that confirmed the STAT as a measure of " $\mathrm{g}$ ". They administered the STAT among 356 psychology students from two universities, and then investigated several models of intelligence. Results of the confirmatory and exploratory factor analyses showed that the "g" model has the best fit, which led to the conclusion that the STAT is a measure of " $\mathrm{g}$ ".

The theory of successful intelligence has been widely used in the Arab world. Recently, Arab researchers have been using the STAT, which has been translated to Arabic 10 years ago as a popular scale around the world, to estimate triarchic abilities. These abilities have not been investigated using advanced statistical methods as most Arab researchers would only report the Cronbach's alpha and total item correlations. The present study was designed to investigate the construct validity of the Arabic version of STAT (Level-H), in addition to gathering evidence of whether STAT should be considered as a measure of " $g$ " or as a measure of triarchic abilities, in the context of the Arab culture, which would be investigated for the first time.

\section{Research method}

\subsection{Participants}

The present study involved the participation of 283 undergraduate male students, with an average age of 19.57 ( $\mathrm{sd}=1.48)$. They were studying special education at the Qassim University in Saudi Arabia for the academic year of 2016/2017 and participated on voluntary basis.

\subsection{Stenberg triarchic abilities test (STAT [Level- H])}

The participants of the present study were subjected to the Sternberg Triarchic Abilities Test (Level-H) which was translated into Arabic by Fadli (2008). The STAT contained nine parts, which were designed to estimate three abilities, namely, analytical, creative, and practical abilities. Each part has twelve items to measure verbal, quantitative, and figural contents, sequentially with three items to measure each part of the content. There were a total of 36 multiple choice items.

\subsection{Research procedure}

The STAT was administered on the study participants, which took approximately 1.2 hours to be completed.

\section{Research findings}

\subsection{Basic statistics}

Total scores for the three abilities were computed. Then, the means and standard deviations were extracted as shown in Table 1 . The results illustrated that the sample performance in terms of analytical ability was the best. These could be explained by the nature of teaching methods, assessment methods, and the emphasis placed on the curriculum related to the analytical ability. Meanwhile, lack of concentration still exists in the practical and creative aspects of the Arab world.

Table 1: Basic statistics for three aspects of intelligence

\begin{tabular}{cccc}
\hline & Analytical & Practical & Creative \\
\hline Mean & 8.34 & 7.72 & 7.21 \\
SD & 3.17 & 3.02 & 3.33 \\
\hline
\end{tabular}

The means and standard deviations of each content score were also calculated as shown in Table 2. The results illustrated that the students' performances were the best and the most consistent in analytical verbal items. These could be explained by the high capacity and interest among the Arab students in the Arabic language as the language of Qur'an. In the Kingdom of Saudi Arabia (KSA), the Arabic language has a distinctive place in the curriculums, from the lower until the upper grades. At least two classes are held every day to learn 
Arabic and its contents due to its uniqueness as a language.

The means and standard deviations of each item were also calculated as shown in Table 3 for further clarifications of STAT. for example: CQ2 (the second item in creative quantitative) had the smallest mean as compared to other items for the same ability and content which may cause by use rational number instead of integer numbers as in other items.

Table 2: Statistics for each content score

\begin{tabular}{|c|c|c|c|c|c|}
\hline & AV & $\mathrm{AQ}$ & $\mathrm{AF}$ & $\mathrm{PV}$ & $P Q$ \\
\hline Mean & 2.98 & 2.61 & 2.75 & 2.69 & 2.53 \\
\hline \multirow[t]{4}{*}{ SD } & 1.07 & 1.40 & 1.24 & 1.09 & 1.29 \\
\hline & $\mathrm{PF}$ & $\mathrm{CV}$ & CQ & $\mathrm{CF}$ & \\
\hline & 2.50 & 2.48 & 2.63 & 2.10 & \\
\hline & 1.19 & 1.26 & 1.30 & 1.45 & \\
\hline $\begin{array}{l}\text { AV: A } \\
\text { Figural; } \\
\text { Figural }\end{array}$ & $\begin{array}{l}\text { l Verk } \\
\text { actical } \\
\text { eative }\end{array}$ & $\begin{array}{r}\text { : Analy } \\
\text { l; PQ: P } \\
\text { l; CQ: } C \\
\text { Figu }\end{array}$ & $\begin{array}{l}\text { uantit } \\
\text { al Quar } \\
\text { e Quan }\end{array}$ & $\begin{array}{l}\text { AF: Ana } \\
\text { ye; } P F \text { : } \\
\text { e; } C F \text { : }\end{array}$ & $\begin{array}{l}\text { al } \\
\text { tical } \\
\text { tive }\end{array}$ \\
\hline
\end{tabular}

Table 3: Statistics for STAT items

\begin{tabular}{cccccccc}
\hline AV1 & AV2 & AV3 & AV4 & AQ1 & AQ2 & AQ3 & AQ4 \\
\hline .74 & .68 & .83 & .74 & .57 & .67 & .65 & .73 \\
.44 & .47 & .38 & .44 & .50 & .47 & .48 & .45 \\
\hline AF1 & AF2 & AF3 & AF4 & PV1 & PV2 & PV3 & PV4 \\
\hline .77 & .63 & .74 & .60 & .69 & .70 & .62 & .68 \\
.42 & .48 & .44 & .49 & .46 & .46 & .49 & .47 \\
\hline PQ1 & PQ2 & PQ3 & PQ4 & PF1 & PF2 & PF3 & PF4 \\
\hline .60 & .61 & .63 & .68 & .51 & .73 & .66 & .60 \\
.49 & .49 & .48 & .47 & .50 & .44 & .47 & .49 \\
\hline CV1 & CV2 & CV3 & CV4 & CQ1 & CQ2 & CQ3 & CQ4 \\
\hline .83 & .48 & .62 & .55 & .72 & .49 & .78 & .65 \\
.38 & .50 & .49 & .50 & .45 & .50 & .42 & .48 \\
\hline CF1 & CF2 & CF3 & CF4 & & & & \\
\cline { 1 - 3 } .51 & .63 & .48 & .48 & & & & \\
.50 & .48 & .50 & .50 & & & & \\
\hline
\end{tabular}

\subsection{Reliability}

The values of alpha coefficient were computed for each process score. The overall scale, as shown in Table 4, illustrated that the STAT has a moderate value of reliability indices.

Table 4: Alpha coefficient values for STAT processes

\begin{tabular}{ccccc}
\hline \multirow{2}{*}{ Alpha } & Analytical & Practical & Creative & Scale \\
\cline { 2 - 5 } & 0.82 & 0.77 & 0.82 & 0.78 \\
\hline
\end{tabular}

A comparison of the reliability values for the STAT processes in the Arabian population was conducted with the results obtained by Sternberg et al. (2001b) over three Western populations, which are shown in Table 5.

Table 5: Alpha coefficient values for the STAT processes

\begin{tabular}{ccccc}
\multicolumn{5}{c}{ among four populations } \\
\hline \multirow{3}{*}{ Alpha } & KSA & U.S. & Finland & Spain \\
\cline { 2 - 5 } & 0.78 & 0.82 & 0.67 & 0.82 \\
\hline
\end{tabular}

Correlations: The correlations between STAT and the abilities it tested were computed as shown in Table 6.

The correlations showed that there was no significant relationship between the three triarchic abilities, which confirmed that each ability represents a unique component that differs from each other. The correlations also showed a moderate and significant relationship $(\mathrm{p}<0.01)$ with the total score of the STAT, which proved its consistency.

Table 6: The correlations between STAT and the intelligence abilities

\begin{tabular}{ccccc} 
& Analytical & Practical & Creative & STAT \\
\cline { 2 - 5 } Analytical & 1.00 & -0.07 & 0.09 & 0.57 \\
Practical & & 1 & 0.11 & 0.55 \\
Creative & & & 1 & 0.69 \\
STAT & & & & 1 \\
\hline
\end{tabular}

\subsection{Confirmatory factor analysis}

Numerous goodness of fit indices were suggested for the assessment of model fit in the CFA. Some researchers might consider Chi-square index $\left(\chi^{2}\right)$ as the most common one, which expresses the difference between the experimental distribution and the observed distribution, depending on a certain zero hypothesis. Within the context of the CFA, this difference should be small enough to accept the expected factorial structure and therefore, the generalized results of the population. In other words, the value of $\left(\chi^{2}\right)$ must be statistically insignificant, which refers to model fit (Hayduk et al., 2007). It may be difficult to achieve this requirement in practice; in the case of a large sample size, small differences may appear statistically significant, indicating a 'Poor Fit'. Some researchers reported that with large samples, the $\left(\chi^{2}\right)$ index could be considered as unaccountable if the scale has good reliability indices (MacCallum, 2003).

However, other researchers tend to use the ratio of Chi-square to the degree of freedom $\left(\chi^{2} / \mathrm{df}\right)$ because each nominator and denominator would increase when the number of parameters is increased. Nonetheless, there is no definitive agreement on the specific values since most researchers would suggest that the value of does not exceed 2 (Gefen et al., 2011). Thus, to avoid the various problems associated with $\left(\chi^{2}\right)$, various goodness of fit indices were suggested within the context of confirmatory factor analysis, such as the Root Mean Square Error of Approximation (RMSEA), the Normed Fit Index (NFI), the Comparative Fit Index (CFI), the Goodness of Fit Index (GFI), and the Incremental Fit Index (IFI).These indices, in addition to another GOF index, were used in the present study to assess model fit.

All models in the present study were analyzed using the maximum likelihood method. Univariate normality was verified by checking the values of kurtosis and skewness and their critical ratios. All values were within the acceptable threshold, according to Kline (2015), as the absolute skew index was $<3$, and the absolute kurtosis index was < 8. Therefore, it can be assumed that no significant violations of the univariate normality existed. Multivariate normality was checked using Mardia's coefficient, which is available in Amos, where it has an acceptable value of $<5$. Additionally, no multivariate outliers were detected by the Mahalanobis distance (i.e., p1 values> 0.001) (Pablos, 2015). The present study investigated three 
models that have been previously tested by Sternberg et al. (2001a;b). The difference with the present study is that parcels were used instead of single items. Each parcel represented the total score for the content (verbal, quantitative, and figural) of each ability (analytical, creative, and practical).

Therefore, it did not assess the rest of Sternberg's models, which are not suitable for parcel cases. Item parcels have been widely used in psychology by numerous researchers who confirmed the advantages of using a few number of parcels in place of large numbers of items. These advantages include better normality approximation, better reliability indices for the parcels' scores, prop goodness of fit, fewer numbers of parameters to be estimated, and a simple process of result interpretations (Bandalos and Fenny, 2001; Marsh and O'Neill, 1984; Marsh et al., 1998; Williams and O'Boyle, 2008; Yang et al., 2010).

Parcels were used in the present study upon checking the factor structure, which verified that no cross loaded items were present. The absence of large sample variability was confirmed and the relation between factors was defined, as confirmed by Marsh et al. (2013). The present study investigated three theoretical models for triarchic intelligence:

- Model A: a general factor model with nine parcels $(\mathrm{AV}, \mathrm{AQ}, \mathrm{AF}, \mathrm{CA}, \mathrm{CQ}, \mathrm{CF}, \mathrm{PA}, \mathrm{PQ}$, and PF).
- Model B: three correlated factors, (i) analytical; (ii)creative; and (iii)practical, with three parcels for each factor, (i) AV, AQ, and AF; (ii) CA, CQ, and $\mathrm{CF}$; and (iii) PA, PQ, and PF, respectively.

- Model C: three uncorrelated factors with three parcels for each factor (consisted of the same variables as in Model 2 minus the correlations).

\subsection{Model fit}

The Amos Graphics was used to analyze the STAT data. The results of the path analyses using parcels instead of individual items have provided important information, which clarified previous findings. The path diagram (Fig. 1) for Model A showed a highly significant path coefficient between the general factor and the analytical content, while the path diagram for Model B (Fig. 2) showed a weak insignificant covariance between the three triarchic abilities.

The present study also used the Amos Graphics to analyze and assess the goodness of fit for these three tested models. The sample size was considered as suitable (Bentler and Chou, 1987). Model fit was assessed using absolute fit indices, relative fit indices, and noncentrality-based indices. Table 7 shows the goodness of fit indices for the three models (A, B, and C).

Table 7: Goodness of fit indices for three models: A, B, and C

\begin{tabular}{ccccc}
\hline Goodness of Fit Index & Criteria & Model (A) G & Model (B) 3-correlated & Model (C) 3-uncorrelated \\
\hline$\chi^{2}$ & Smallest & 554.47 & 48.49 & 54.65 \\
$\mathrm{p}-$ value & p-value $>0.05$ & 0.000 & 0.002 & 0.001 \\
$\chi^{2} / d f$ & $\chi^{2} / d f \leq 5$ & 20.54 & 2.02 & 2.02 \\
IFI & IFI $\geq 0.95$ & 0.38 & 0.97 & 0.97 \\
CFI & CFI $\geq 0.95$ & 0.37 & 0.97 & 0.97 \\
NFI & NFI $\geq 0.95$ & 0.37 & 0.94 & 0.93 \\
AGFI & AGFI $\geq 0.80$ & 0.49 & 0.93 & 0.93 \\
GFI & GFI $\geq 0.80$ & 0.69 & 0.96 & 0.96 \\
TLI & TLI $\geq 0.90$ & 0.16 & 0.96 & 0.06 \\
RMSEA & RMSEA $\leq 0.06$ & 0.27 & 0.06 & 90.65 \\
AIC & Smallest & 590.47 & 90.49 & 156.27 \\
BIC & difference than 10 in -ve & 656.09 & 167.05 & 27 \\
$d f$ & & 27 & 24 & \\
\hline
\end{tabular}

Since the tested models were nested, the fit indices, TLI $>0.9$ and RMSEA $<0.05$, were used to assess model fitness. Chi square statistics showed that the smaller value showed a better fit, while $\mathrm{p}$ values $<0.05$ were undesirable .However, in the case of a large sample size, these values can be ignored. To compare the nested models, the Bayesian Information Criterion (BIC) and the Akaike's Information Criterion (AIC) were used, where a difference of 10 is considered as a better fit with a smaller value of BIC (Hu and Bentler, 1999).

Numerous confirmatory factor analyses were conducted to assess model fit for the three models. Model A was a general factor model with 12 parcels, with each parcel representing the content of each ability.

Model B was a more complicated model, which consisted of three main correlated abilities (Triarchic intelligences), and each ability consisted of three parcels, with each parcel representing the total score for each content (Verbal, Quantitative, and Figural).

Meanwhile, Model C was similar to Model B, but its abilities were uncorrelated. The results were somewhat similar to the findings of Sternberg et al. (2001b) and Chooi et al. (2014) for Models B and C both models showed good fit and fairly similar values of fit indices. However, according to the BIC and AIC indices, Model $\mathrm{C}$ was considered as having the better fit. Unlike the results obtained by Sternberg et al. (2001a) and Chooi et al. (2014), Model A with the general factor was far enough from the other models and it has unacceptable values for all fit indices. Thus, the present study concluded that the triarchic intelligence, which can be assessed using the STAT, cannot be considered in any way, as a measure of general factor as previously claimed. 


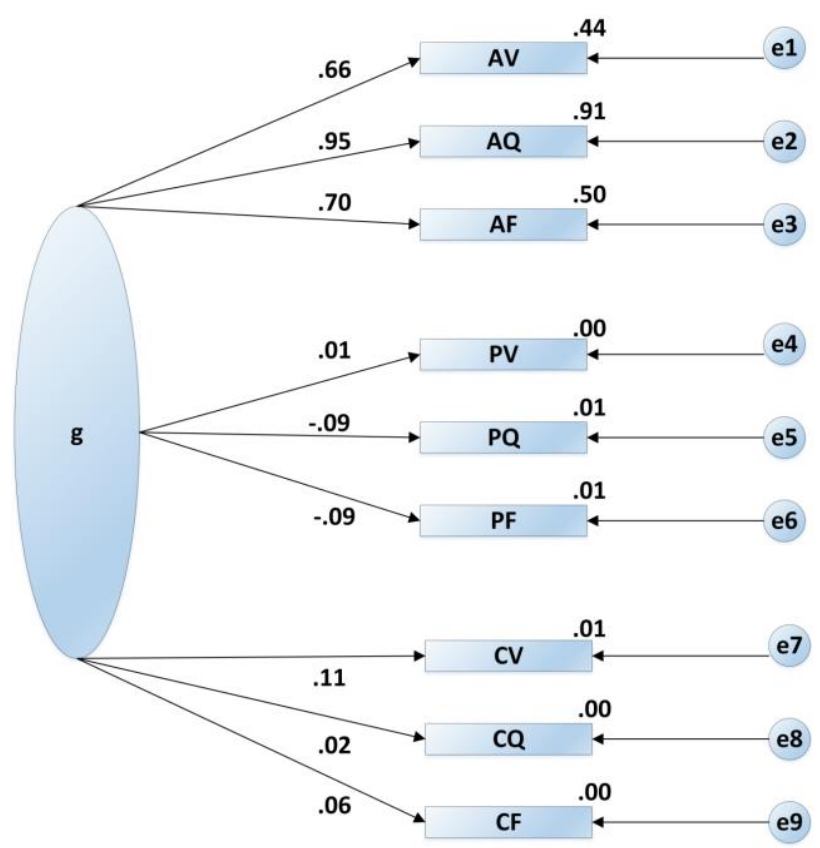

Fig. 1: Model A (g factor with 9 parcels)

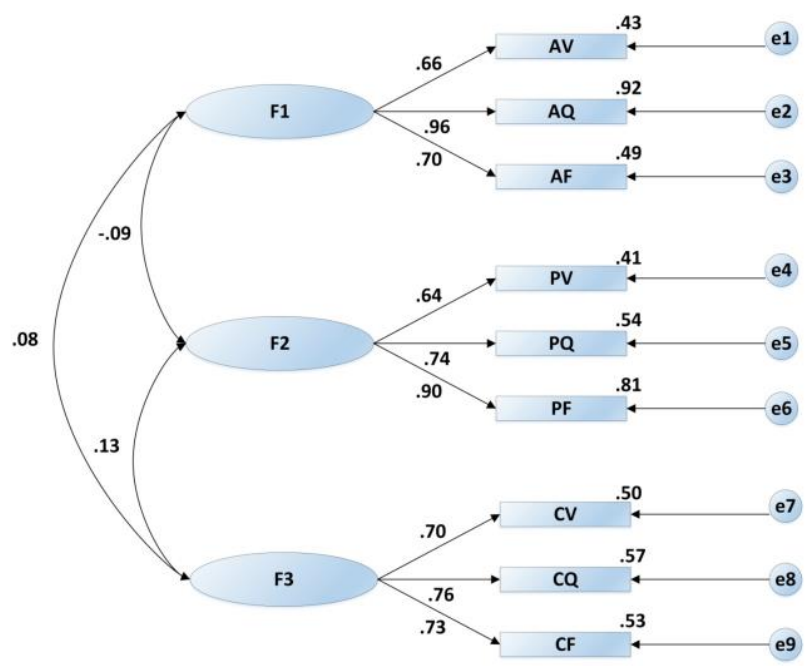

Fig. 2: Model B (three correlated abilities)

\section{Discussion}

The results of the present study, which depended on the confirmatory factor analysis, have supported the structural validity of the STAT. These results have provided an indirect empirical evidence of the triarchic intelligence theory. The procedures applied in the present study allowed comparisons to be made between three different models. However, for the first time, parcels were used instead of individual items because of their advantages in previous studies (Bandalos and Fenny, 2001; Marsh and O'Neill, 1984; Marsh et al., 1998; Williams and O’Boyle, 2008; Yang et al., 2010). Several analyses were conducted in the present study, which included correlations and confirmatory factor analysis. In general, the results showed an absence of the traditional concept of intelligence, known as the " $\mathrm{g}$ " factor.

Table 6 shows that there were modest correlations (0.55-0.69) between the total score of the STAT and each individual ability (analytical, practical, and creative), as there were no significant correlations between the three abilities. In addition, a negative correlation was observed between the analytical and practical abilities. These findings confirmed Sternberg's statements that the three triarchic abilities are separated, but could be correlated (Sternberg, 2003).

Nonetheless, these results were slightly different than the results Sternberg obtained in his research. These differences could be caused by cultural effects, language, and lifestyle in Asian cultures, especially in the Arab culture, which has a unique social nature. Previous researchers have reported that the concept of intelligence in many Asian cultures places more emphasize on the social aspect of intelligence compared to in Western cultures (Azuma and Kashiwaga, 1987; Lutz, 1985; Poole, 1985; White, 1985).

The negative correlation between practical and analytical abilities has also confirmed Sternberg's statements, whereby practical abilities could not correlate with analytical abilities (Sternberg et al., 2000; Sternberg et al., 2001a). This result can be caused by the clear difference, for example, between being intelligent on paper, and yet, unsuccessful in daily life. The results shown by the path analyses for the STAT parcels offered more information about this test. The results in Fig. 1 showed highly significant path coefficients between the three parcels of analytical abilities and the " $g$ " factor, which could explain the findings of previous researches (Brody 2003; Chooi et al., 2014; Gottfredson, 2003), and Analytical ability could be considered as synonymous with the traditional " $\mathrm{g}$ " intelligence because of its various aspects, namely, the ability to analyze, evaluate, critique, and compare contrasting things, which can all be measured using conventional academic tests.

The results of the path analysis shown in Fig. 2 have confirmed that highly significant path coefficients existed between each content parcel and each ability. The path coefficients between the analytical parcels and the three abilities have the smallest values, which supported the Triarchic Theory as a distinct concept of " $g$ " factor intelligence. The results have also shown trivial covariance between the three triarchic abilities, which confirmed them to be separate constructs. The results of the path analysis, as shown in Fig. 3, indicated that the quantitative content was the best predictor for each analytical and creative ability. Meanwhile, the figural content was the best predictor for creative ability, which may provide some information for the development of triarchic intelligence tests in the future.

To replicate the findings obtained by Sternberg et al. (2001a), several structural equation models were employed. The model that has the best fit was the model of three uncorrelated abilities, which was assigned as Model C Minor differences were observed between this model and the model of three correlated abilities, which was assigned as Model B. 
Although both Models B and C have acceptable goodness of fit indices, a difference of 10.78 of BIC value suggested that Model $\mathrm{C}$ was the best fit model. This triarchic model was not the same model that Chooi et al. (2014) found to be the best fit model, which exactly confirmed the triarchic intelligence theory.
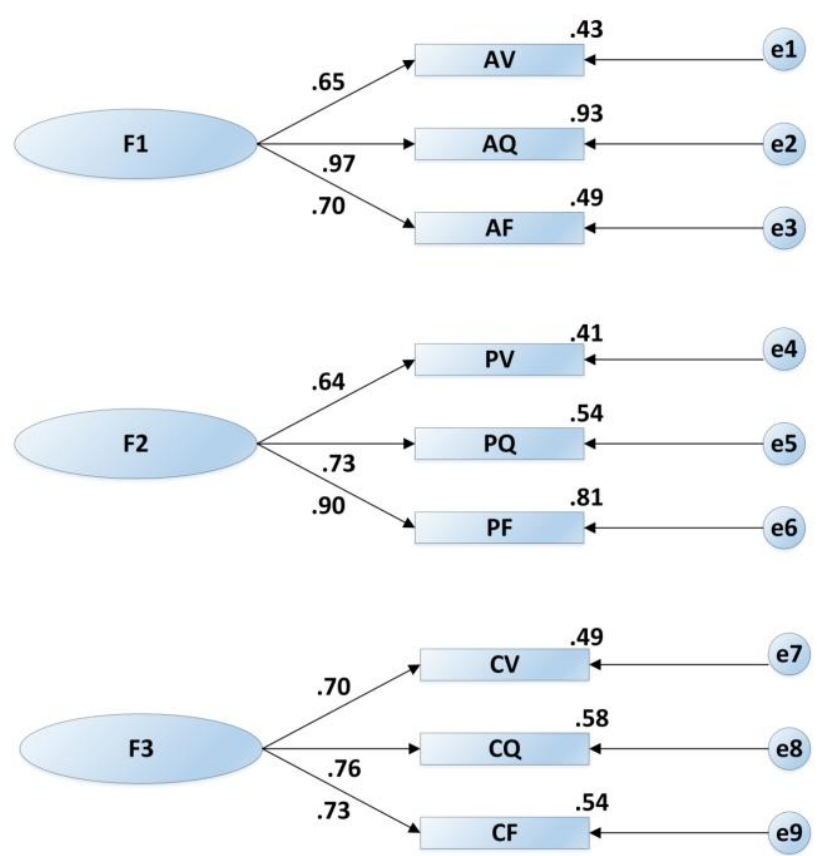

Fig. 3: Model C (three uncorrelated abilities)

Unlike many other research findings (Brody, 2003; Chooi et al., 2014; Gottfredson, 2003), results of the present study showed that the model of the general intelligence factor has a lack of fit. This was far enough from Models B and C with their three abilities. Hence, it could be concluded that the STAT is a good measure of triarchic intelligence.

\section{Research limitations and future direction}

The main limitations faced by the present study are as follows. First, the study sample consisted of only male students. This could affect the results because male brains tend to perform tasks predominantly on the left side, which is the logical or rational side of the brain.

Secondly, the present study only gave information about the nature of triarchic intelligence in the Arab culture, but failed to correlate it with other intelligence tests for better clarification and confirmation of its structure. Further research of the psychometric properties of the STAT, over both males and females, should be conducted using item response theory models, and the differential functioning of the STAT items over these models.

\section{Conflicting interests}

The author(s) declared no potential conflicts of interest with respect to the research, authorship, and/or publication of this article.

\section{Funding}

The author(s) received no financial support for the research, authorship, and/or publication of this article.

\section{References}

Azuma H and Kashiwagi K (1987). Descriptions for an intelligent person: A Japanese study. Japanese Psychological Research, 29(1): 17-26.

Bandalos DL and Finney J (2001). Item parceling issues in structural equation modeling. In: Marcoulides GA and Shumaker RE (Eds.), Advanced structural equation modeling: New developments and techniques: 269-296. Lawrence Erlbaum Associates, Mahwah, USA.

Bentler PM and Chou CP (1987). Practical issues in structural modeling. Sociological Methods and Research, 16(1): 78-117.

Berry JW (1974). Radical cultural relativism and the concept of intelligence. In: Berry JW and Dasen PR (Eds.), Culture and cognition: Readings in cross-cultural psychology: 225-229. Methuen, London, UK.

Brody $N$ (2001). Inspection time: Past, present, and future. Intelligence, 29(6): 537-542.

Brody N (2003). Construct validation of the Sternberg triarchic abilities test comment and reanalysis. Intelligence, 31(4): 319329.

Chooi WT, Long LE, and Thompson A (2014). The sternberg triarchic abilities test (Level-H) is a measure of G. Journal of Intelligence, 2(3): 56-67.

Ekinci B (2014). The relationships among Sternberg's triarchic abilities, gardner's multiple intelligences, and academic achievement. Social Behavior and Personality, 42(4): 625-633.

Fadli F (2008). The relationship between three dimensional intelligence and teenager students: Meta-cognition process within demographic changes in the state of Kuwait. Ph.D Dissertation, Amman Arab University, Jordan, Amman.

Gefen D, Rigdon E, and Straub D (2011). An update and extension to SEM guidelines for administrative and social science research. MIS Quarterly. 35(2): 3-14.

Gottfredson LS (2003). Dissecting practical intelligence theory: Its claims and evidence. Intelligence, 31(4): 343-397.

Grigorenko EL and Sternberg RJ (2001). Analytical, creative, and practical intelligence as predictors of self-reported adaptive functioning: A case study in Russia. Intelligence, 29(1): 57-73.

Hayduk L, Cummings G, Boadu K, Pazderka-Robinson H, and Boulianne S (2007). Testing! Testing! One, two, three: Testing the theory in structural equation models. Personality and Individual Differences, 42(5): 841-850.

Hu LT and Bentler PM (1999). Cut off criteria for fit indexes in covariance structure analysis: conventional criteria versus new alternatives. Structural Equation Modeling, 6(1): 1-55.

Kaufman SB and Singer JL (2004). Applying the theory of successful intelligence to psychotherapy training and practice. Imagination, Cognition, and Personality, 23(4): 325-355.

Kline RB (2015). Principles and practice of structural equation modeling. Guilford Publications, New York, USA.

Lutz C (1985). Ethnopsychology compared to what? Explaining behavior and consciousness among the Ifaluk. In: White GM and Kirkpatrick J (Eds.), Person, self, and experience: Exploring pacific ethnopsychology: 35-79. University of California Press, Berkeley, USA.

MacCallum RC (2003). 2001 presidential address: working with imperfect models. Multivariate Behavioral Research, 38(1): 113-139. 
Marsh HW and O'Neill R (1984). Self-description questionnaire III (SDQ III): The construct validity of multidimensional selfconcept ratings by late-adolescents. Journal of Educational Measurement, 21(2): 153-174.

Marsh HW, Hau KT, Balla JR and Grayson D (1998). Is more ever too much? The number of indicators per factor in confirmatory factor analysis. Multivariate Behavioral Research, 33(2): 181-220.

Marsh HW, Lüdtke O, Nagengast B, and Morin AJS (2013). Why item parcels are (almost) never appropriate: two wrongs do not make a right camouflaging misspecification with item parcels in CFA models. Psychological Methods, 18(3): 257284.

Pablos PO (2015). Technological solutions for sustainable business practice in Asia. IGI Global, Hershey, USA.

Poole FJP (1985). Coming in to social being: Cultural images of infants in Bimin-Kuskusmin folk psychology. In: White GM and Kirkpatrick J (Eds.), Person, self, and experience: Exploring pacific ethnopsychology: 183-242. University of California Press, Berkeley, USA.

Ruzgis P and Grigorenko E (1994). Cultural meaning systems, intelligence, and personality. In: Sternberg RJ and Ruzgis P (Eds.), Personality and Intelligence: 248-270. Cambridge University Press, Cambridge, UK.

Sternberg R (1997). The concept of intelligence and its role in lifelong learning and success. American Psychologist, 52(10): 1030-1037.

Sternberg R (2003). A broad view of intelligence: The theory of successful intelligence. Consulting Psychology Journal: Practice and Research, 55(3): 139-154.
Sternberg R and Grigorenko E (2006). Cultural intelligence and successful intelligence. Group and Organization Management, 31(1): 27-39.

Sternberg R, Castejon J, Prieto M, Hautamäki J, and Grigorenko E (2001a). Confirmatory factor analysis of the Sternberg Triarchic Abilities Test in three international samples: an empirical test of the triarchic theory of intelligence. European Journal of Psychological Assessment, 17(1): 1-16.

Sternberg R, Forsythe G, Horvath J, Hedlund J, Snook S, Williams W, and Grigorenko E (2000). Practical intelligence in everyday life. Cambridge University Press, Cambridge, UK.

Sternberg R, Grigorenko E, Ferrari M, and Clinkenbeard P (1999). A triarchic analysis of an aptitude-treatment interaction. European Journal of Psychological Assessment, 15(1): 3-13.

Sternberg RJ, Grigorenko E, and Bundy DA (2001b). The predictive value of IQ. Merrill-Palmer Quarterly, 47(1): 1-41.

White G (1985). Premises and purposes in a Solomon Island ethnopsychology. In: White GM and Kirkpatrick J (Eds.), Person, self, and experience: Exploring pacific etnopsychology: 328-366. University of California Press, Berkeley, USA.

Williams L and O'Boyle E (2008). Measurement models for linking latent variables and indicators: A review of human resource management research using parcels. Human Resource Management Review, 18(4): 233-242.

Yang C, Nay S, and Hoyle R (2010). Three approaches to using lengthy ordinal scales in structural equation models: Parceling, latent scoring, and shortening scales. Applied Psychological Measurement, 34(2): 122-142.

Yang SY and Sternberg RJ (1997). Taiwanese Chinese people's conceptions of intelligence. Intelligence, 25(1): 21-36. 